\title{
Fermi surfaces and Luttinger's theorem in paired fermion systems
}

\section{Citation}

Sachdev, Subir, and Kun Yang. 2006. "Fermi Surfaces and Luttinger's Theorem in Paired Fermion Systems." Physical Review B 73 (17). https://doi.org/10.1103/physrevb.73.174504.

\section{Permanent link}

http://nrs.harvard.edu/urn-3:HUL.InstRepos:41417239

\section{Terms of Use}

This article was downloaded from Harvard University's DASH repository, and is made available under the terms and conditions applicable to Other Posted Material, as set forth at http:// nrs.harvard.edu/urn-3:HUL.InstRepos:dash.current.terms-of-use\#LAA

\section{Share Your Story}

The Harvard community has made this article openly available.

Please share how this access benefits you. Submit a story.

\section{Accessibility}




\title{
Fermi surfaces and Luttinger's theorem in paired fermion systems
}

\author{
Subir Sachdev and Kun Yang* \\ Department of Physics, Harvard University, Cambridge, Massachusetts 02138, USA
}

(Dated: June 8, 2018)

\begin{abstract}
We discuss ground state properties of a mixture of two fermion species which can bind to form a molecular boson. When the densities of the fermions are unbalanced, one or more Fermi surfaces can appear: we describe the constraints placed by Luttinger's theorem on the volumes enclosed by these surfaces in such Bose-Fermi mixtures. We also discuss the nature of the quantum phase transitions involving changes in the number of Fermi surfaces.
\end{abstract}

\section{INTRODUCTION}

Recent experiments [1, 2] on trapped ultracold atoms have refocused theoretical attention on pairing between distinct fermion species, in situations in which the densities of the two species are unequal. In these experiments unequal numbers of two hyperfine states of fermionic ${ }^{6} \mathrm{Li}$ atoms were mixed and scanned across a Feshbach resonance. On one side of the resonance the fermions bind to form a bosonic molecule which can condense into a BoseEinstein condensate (BEC), while on the other side they experience a weak attractive interaction which leads to formation of Cooper pairs in a Bardeen-Cooper-Schrieffer (BCS) state. When the densities of the hyperfine species are unbalanced, some fermions are unpaired, and this raises the possibility of co-existence of a bosonic condensate and one or more Fermi surfaces in the ground state.

This paper will describe the constraints that Luttinger's theorem places on the volumes enclosed by Fermi surfaces under such conditions. Such constraints are distinct in different phases, and we will also describe the quantum phase transitions across which the statement of Luttinger's theorem changes. We note that these Fermi surface volume constraints are exact, and apply even in strongly interacting regimes where the fermions fluctuate into bosonic molecular states at short scales. In such situations short-range spectroscopic observables may indicate that fermions exist in molecules, but the true ground state will nevertheless have the undiminished Fermi surface volume(s), albeit with a reduced quasiparticle residue at the Fermi surface. Our results indicate that the volumes of the Fermi surfaces are sensitive to the presence or absence of a Bose condensate of the molecules; thus they can also be used to probe superfluidity or pair coherence. In principle the Fermi surface volumes can be measured from the momentum distributions of the atoms. Such a measurement was recently performed in a gas of ${ }^{40} \mathrm{~K}$ across a Feshbach resonance 3 . In this experiment the effect of the trap on the momentum distribution appears to be quite strong, such that the discontinuity in momentum distribution gets wiped

\footnotetext{
* On leave from Department of Physics, Florida State University,
} Tallahassee, FL 32306. out even for non-interacting fermions. We hope that by manipulating the form of the trap potential, its effect can be minimized so that discontinuities in momentum distribution associated with Fermi surfaces can be detected in future experiments; this would probably require a trap potential that is flat inside the trap and rises very fast near the boundary.

We note that Luttinger's theorem for Bose-Fermi mixtures has also been investigated in recent work [4, 5] in which a boson and a fermion bind to form a fermionic molecular state. Here we will show that these results can be straightforwardly extended to the case of interest to the recent atomic experiments: two fermions binding to form a bosonic molecular state. We also use nonperturbative arguments similar to those of Yamanaka, Oshikawa and Affleck [6] to establish analogous results in one dimension.

\section{HAMILTONIAN AND PHASE DIAGRAM}

Our results are rather general, and apply to a wide class of Hamiltonians of the form

$$
\begin{gathered}
H=H_{f}+H_{b}+\sum_{\mathbf{k}, \mathbf{k}^{\prime}}\left(V_{\mathbf{k}, \mathbf{k}^{\prime}} f_{\uparrow, \mathbf{k}}^{\dagger} f_{\downarrow, \mathbf{k}^{\prime}}^{\dagger} b_{-\mathbf{k}-\mathbf{k}^{\prime}}+h . c .\right) \\
-\sum_{\mathbf{k}}\left((\mu+h) f_{\uparrow, \mathbf{k}}^{\dagger} f_{\uparrow, \mathbf{k}}+(\mu-h) f_{\downarrow, \mathbf{k}}^{\dagger} f_{\downarrow, \mathbf{k}}\right. \\
\left.+(2 \mu-\nu) b_{\mathbf{k}}^{\dagger} b_{\mathbf{k}}\right)
\end{gathered}
$$

where $f_{\uparrow, \downarrow}$ are annihilation operators of the two fermion species, $b$ is the annihilation operator of the bosonic molecule, $H_{f}$ and $H_{b}$ involve fermionic and bosonic operators only (which contain their kinetic energies and may also include additional interactions). The fermion species are at chemical potentials $\mu_{f \uparrow}=\mu+h$ and $\mu_{f \downarrow}=\mu-h$, while the boson is at chemical potential $\mu_{b}=2 \mu-\nu$. Here $h$ is the 'field' which unbalances the fermion densities, and $\nu$ is the 'detuning' across the Feshbach resonance which scans between the BCS and BEC limits.

It is useful to frame our discussion using a ground state phase diagram as a function of $\nu$ and $h$. Such a phase diagram has been studied in a number of recent works 7, 8, 9, 10, 11, 12], and it is not our purpose here to critique these results. Rather we find it useful to work with the schematic phase diagram shown in Fig. 1] which 


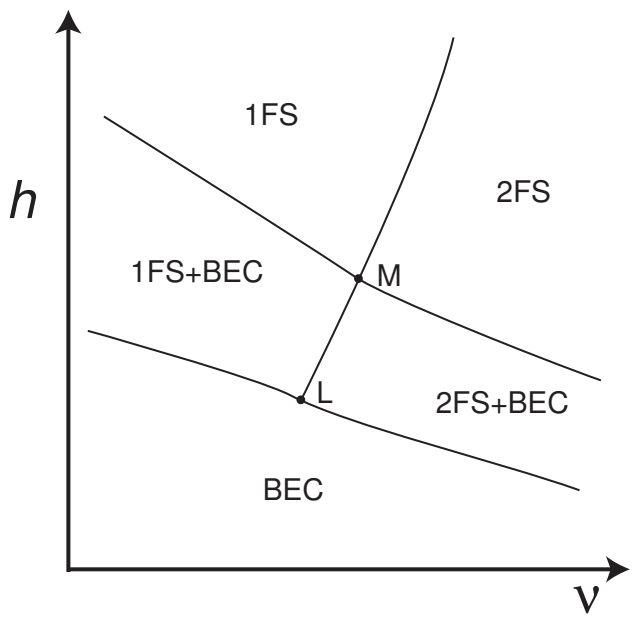

FIG. 1: Schematic zero temperature phase diagram as a function of the 'field' $h$ and the detuning $\nu$. The phases labeled $\mathrm{BEC}$ have $\langle b\rangle \neq 0$, and include regions where the pairing is more properly considered to be of the BCS type. States labeled $n$ FS have $n$ Fermi surfaces. Only homogeneous, translationally invariant states are shown, and possible instabilities to phase separation and modulated FFLO states have been omitted. Two Luttinger theorems, in Eq. (13), apply in the phases without the BEC. The phases with the BEC have only one Luttinger theorem, specified in Eq. (12).

omits instabilities towards phase separation and broken translational invariance. As we will discuss later, our results are easily extended to modulated phases like the Fulde-Ferrell-Larkin-Ovchinnikov (FFLO) state [13, 14].

It is easiest to first understand the phases at small and large $h$ in Fig 1 At small $h$ we have the conventional paired fermion state with equal fermion densities, $N_{\uparrow}=N_{\downarrow}$, where $N_{\uparrow}=\sum_{\mathbf{k}} f_{\uparrow \mathbf{k}}^{\dagger} f_{\uparrow, \mathbf{k}}$ and similarly for $N_{\downarrow}$; this interpolates from the BEC to the BCS limits with increasing $\nu$. At very large $h$, we have conventional Fermi liquid states with no BEC, $\langle b\rangle=0$; these can have a single Fermi surface of $f_{\uparrow}$ alone with $N_{\downarrow}=0$, or two Fermi surfaces with one each for $f_{\uparrow}$ and $f_{\downarrow}$, and both $N_{\uparrow}, N_{\downarrow}$ non-zero.

At intermediate $h$, we have the possibility of phases with co-existence of the BEC and Fermi surface(s). These can be understood most simply by adding a non-zero $\langle b\rangle$ to the effective Hamiltonian for the large $h$ Fermi liquid states. The $2 \mathrm{FS}+\mathrm{BEC}$ phase is known as the Sarma 15] or "breached pair" [16] state. This intermediate $h$ region is most likely to be susceptible to further instabilities towards phase separation [17] and modulated phases (which we will note later) in the weak coupling (large positive $\nu$ ) regime. There is numerical evidence suggesting it may become stable in the intermediate and strong coupling regimes [18].

\section{LUTTINGER'S THEOREM}

For the decoupled Hamiltonian $H_{f}+H_{b}$, the numbers of the two fermion species are separately conserved, and the original version of the Luttinger's theorem states that there are two Fermi surfaces that correspond to the two fermion species, whose volumes are determined by the numbers of each fermion species. Here we extend the Luttinger's theorem to include the full $H$, which mixes fermions with bosons and break the conservation of the fermion number for individual fermion species. We find that the two Fermi surfaces remain to have separately conserved volumes as long as there is no Bose condensate (or no broken $\mathrm{U}(1)$ symmetry). In the presence of a Bose condensate, the volumes of individual Fermi surfaces are no longer conserved, but we will now show that the difference of the volumes of the two Fermi surfaces remains conserved, and this difference is determined by the density or number difference in $\uparrow$ and $\downarrow$ fermions (which commutes with $H$ ):

$$
\Delta N=N_{\uparrow}-N_{\downarrow}=\sum_{\mathbf{k}}\left(f_{\uparrow, \mathbf{k}}^{\dagger} f_{\uparrow, \mathbf{k}}-f_{\downarrow, \mathbf{k}}^{\dagger} f_{\downarrow, \mathbf{k}}\right) .
$$

As we noted earlier, closely related results were obtained on a different model of Bose-Fermi mixture [4].

We introduce the $2 \times 2$ Green's function matrix for fermions in the usual manner to allow for pairing and appearance of anomalous Green's function:

$$
\hat{G}_{F}(\mathbf{x}, t)=-i\left\langle T\left[f_{\uparrow}(\mathbf{x}, t), f_{\downarrow}^{\dagger}(\mathbf{x}, t)\right]^{T}\left[f_{\uparrow}^{\dagger}(0,0), f_{\downarrow}(0,0)\right]\right\rangle .
$$

$\Delta N$ is related to the Green's function matrix in the following manner:

$$
\begin{aligned}
& \sum_{\mathbf{k}}\left(f_{\uparrow \mathbf{k}}^{\dagger} f_{\uparrow \mathbf{k}}+f_{\downarrow \mathbf{k}} f_{\downarrow \mathbf{k}}^{\dagger}\right)=N_{0}+\Delta N \\
& =\frac{-i A}{(2 \pi)^{d+1}} \int d \omega d^{d} \mathbf{k} e^{i \omega 0^{+}} \operatorname{tr} \hat{G}_{F}(\omega, \mathbf{k}),
\end{aligned}
$$

where $N_{0}=\sum_{\mathbf{k}} 1$ is the total number of single particle states within a high momentum cutoff or a band within which the model is defined, $A$ is the system volume, and $d$ is dimensionality. Using the Dyson's equation for fermion self-energy (including anomalous self-energy that is offdiagonal)

$$
\hat{\Sigma}_{F}(\omega, \mathbf{k})=\omega-\hat{\xi}_{\mathbf{k}}-\hat{G}_{F}^{-1}(\omega, \mathbf{k}),
$$

where $\hat{\xi}_{\mathbf{k}}$ is the (diagonal) kinetic energy matrix for $\uparrow$ fermion and $\downarrow$ hole (each measured from the chemical potential), one may rewrite Eq. (4) as

$$
\begin{aligned}
N_{0}+\Delta N & =\frac{i A}{(2 \pi)^{d+1}} \int d \omega d^{d} \mathbf{k} e^{i \omega 0^{+}} \operatorname{tr}\left[\frac{\partial}{\partial \omega} \log \hat{G}_{F}(\omega, \mathbf{k})\right. \\
& \left.-\hat{G}_{F}(\omega, \mathbf{k}) \frac{\partial}{\partial \omega} \hat{\Sigma}_{F}(\omega, \mathbf{k})\right]
\end{aligned}
$$

The proof of Luttinger's theorem relies on the existence of a Luttinger-Ward (LW) functional $\Phi_{L W}\left[G^{\prime}\right]$, 
whose functional derivative gives the exact self-energy when evaluated at the exact Green's function [19]:

$$
\left.\frac{\delta \Phi_{L W}\left[G^{\prime}\right]}{\delta G^{\prime}}\right|_{G^{\prime}=G}=\Sigma .
$$

Recently it has been shown [20] the LW functional can be constructed non-perturbatively under very general situations, including cases with spontaneously broken symmetry. This is particularly important in the latter cases, as the broken symmetry states are not smoothly connected to non-interacting systems, and the Green's functions may contain "anomalous" terms. In the present case the LW functional is a functional of both fermion and boson Green's functions, and the conservation of $\Delta N$ guarantees that

$\Phi_{L W}\left[\hat{G}_{F}^{\prime}(\omega, \mathbf{k}), G_{B}^{\prime}(\omega, \mathbf{k})\right]=\Phi_{L W}\left[\hat{G}_{F}^{\prime}(\omega+\alpha, \mathbf{k}), G_{B}^{\prime}(\omega, \mathbf{k})\right] ;$

this is a consequence of the invariance of $\Phi_{L W}\left[\hat{G}_{F}^{\prime}(\omega, \mathbf{k}), G_{B}^{\prime}(\omega, \mathbf{k})\right]$ under gauge transformation $f_{\uparrow} \rightarrow f_{\uparrow} e^{i \delta} ; f_{\downarrow} \rightarrow f_{\downarrow} e^{-i \delta}[20]$. It leads to

$$
\frac{\partial \Phi_{L W}\left[\hat{G}_{F}^{\prime}, G_{B}^{\prime}\right]}{\partial \alpha}=\int d \omega d^{d} \mathbf{k} \operatorname{tr}\left[\frac{\delta \Phi_{L W}}{\delta \hat{G}_{F}^{\prime}} \frac{\partial \hat{G}_{F}^{\prime}}{\partial \omega}\right]=0 .
$$

Now use the exact Green's functions as the argument of $\Phi_{L W}$ in the equation above, and generalize Eq. (7) to the matrix form:

$$
\left.\frac{\delta \Phi_{L W}\left[\hat{G}_{F}^{\prime}, G_{B}^{\prime}\right]}{\delta \hat{G}_{F}^{\prime}}\right|_{\hat{G}^{\prime}=\hat{G}}=\hat{\Sigma}_{F}
$$

one can show the last term in Eq. (6) vanishes by combining Eqs. (10) and (9). The manipulation of the remaining term (that involve $\hat{G}_{F}$ only) is straightforward and standard [19, 21]; one may, for example, diagonalize $\hat{G}_{F}$ to perform the trace. This leads to

$$
N_{0}+\Delta N=\frac{A}{(2 \pi)^{d}} \int d^{d} \mathbf{k}\left[\Theta\left(-e_{\uparrow}(\mathbf{k})\right)+\Theta\left(-e_{\downarrow}(\mathbf{k})\right)\right],
$$

where $e_{\uparrow, \downarrow}(\mathbf{k})$ are the eigenvalues of $-\hat{G}^{-1}(\omega=0, \mathbf{k})$. In the absence of interactions $e_{\uparrow, \downarrow}(\mathbf{k})$ are simply the single particle excitation energies, and the k's at which they vanish define the Fermi surfaces. In the presence of interactions there is a self-energy contribution to $e_{\uparrow, \downarrow}(\mathbf{k})$, which in general contains an imaginary part. However for the quasiparticles to be well-defined in the low-energy limit, the imaginary part of the self-energy must vanish for $\omega=0$, so that the quasiparticles are long-lived. As a consequence $e_{\uparrow, \downarrow}(\mathbf{k})$ are real, and are the (interactionrenormalized) quasiparticle excitation energies, and the k's at which they vanish define the location of the Fermi surfaces in the presence of interactions. Thus the two terms on the right hand side of Eq. (11) are the volumes of the two Fermi seas for $\uparrow$ particle-like quasiparticles and $\downarrow$ hole-like quasiparticles; this is because $e_{\uparrow}(\mathbf{k})$ is an increasing function of $|\mathbf{k}|$, while $e_{\downarrow}(\mathbf{k})$ is a decreasing one.
To express results in terms of particle-like Fermi seas, we rewrite Eq. (11) as

$$
\begin{aligned}
\Delta N & =-N_{0}+\frac{A}{(2 \pi)^{d}} \int d^{d} \mathbf{k}\left[\Theta\left(-e_{\uparrow}(\mathbf{k})\right)+1-\Theta\left(e_{\downarrow}(\mathbf{k})\right)\right] \\
& =\frac{A}{(2 \pi)^{d}} \int d^{d} \mathbf{k}\left[\Theta\left(-e_{\uparrow}(\mathbf{k})\right)-\Theta\left(e_{\downarrow}(\mathbf{k})\right)\right] \\
& =\frac{A}{(2 \pi)^{d}}\left(\Omega_{\uparrow}-\Omega_{\downarrow}\right),
\end{aligned}
$$

where we used the fact that $N_{0}=\frac{A}{(2 \pi)^{d}} \int d^{d} \mathbf{k}$. We have thus related the fermion number difference $\Delta N$ to the Fermi surface volumes $\Omega_{\uparrow}, \Omega_{\downarrow}$ in a form analogous to the Luttinger's theorem [21]. The statement in Eq. (12) applies in all the phases of Fig. 11 The BEC phase has no Fermi surface, and so in this phase we must have $N_{\uparrow}=N_{\downarrow}$.

In the absence of Bose condensation, $\hat{\Sigma}$ (and therefore $\hat{G}_{F}$ ) is diagonal in the original basis of $f_{\uparrow, \downarrow}$; in this case one can interpret the two Fermi surfaces as those for the $\uparrow, \downarrow$ fermions respectively. By using the fact that bosonic excitations are gapped in the absence of a Bose condensate [4, one can further prove that their volumes are fixed individually, so that

$$
N_{\uparrow}+N_{B}=\frac{A}{(2 \pi)^{d}} \Omega_{\uparrow} ; \quad N_{\downarrow}+N_{B}=\frac{A}{(2 \pi)^{d}} \Omega_{\downarrow},
$$

where $N_{B}=\sum_{\mathbf{k}} b_{\mathbf{k}}^{\dagger} b_{\mathbf{k}}$. The result (13) applies in all phases of Fig. 1 without a BEC. Among these, the 1FS phase only has a Fermi surface for the $\uparrow$ fermions, and so Eq. (13) implies that in this phase we must have $N_{\downarrow}=0$ and $N_{B}=0$. Note that $N_{\uparrow, \downarrow}$ and $N_{B}$ are not individually conserved, but the combinations on the left-hand-sides of Eq. (13) are conserved in the absence of a Bose condensate. The proof of Eq. (13) parallels the analogous one in Ref. 4.

In our discussion so far we have assumed the system to be uniform. Our results, however, can be generalized to cases with spontaneously broken translational symmetry. An important example of this case is the FFLO superfluid state [13, 14], in which the Bose condensate has a periodic spatial structure. In such cases, the Fermi surface volumes are well-defined modulo the Brillouin zone volume $\Omega_{B}$; as a consequence all of our statements on the constraints on Fermi surface volumes are modulo $\Omega_{B}$. The situation is identical to electrons moving in a periodic potential considered by Luttinger originally [21]. We note in passing that the possible realization of the FFLO state in $\mathrm{CeCoIn}_{5}$ 24, 25] is currently being investigated experimentally.

\section{ONE DIMENSIONAL SYSTEMS}

We now turn our discussion to one-dimensional (1D) systems, where there are no well-defined quasiparticles, but there can still be well-define Fermi wavevectors and 
corresponding Luttinger's theorem [6]. In the following we consider a $1 \mathrm{D}$ lattice version of $H$ :

$$
\begin{aligned}
H_{1 D} & =-t_{f} \sum_{i, \sigma}\left(f_{\sigma i}^{\dagger} f_{\sigma i+1}+h . c .\right)-t_{b} \sum_{i}\left(b_{i}^{\dagger} b_{i+1}+h . c .\right) \\
& -g \sum_{i}\left(f_{\uparrow i}^{\dagger} f_{\downarrow i}^{\dagger} b_{i}+\text { h.c. }\right)+H_{\text {int }},
\end{aligned}
$$

where $i$ is the site index and $H_{\text {int }}$ involves fermion or boson number operators only. Such models have been considered recently [22, 23] in the context of mixture and coherence between fermionic atoms and bosonic molecules in 1D. Here we consider the most generic case that $N_{\uparrow}-N_{\downarrow} \neq 0$ and the particle fillings are incommensurate with the lattice, so that neither spin nor charge gap is possible; generalizations to commensurate cases are straightforward. In the absence of the bosonic degrees of freedom, Eq. (14) describes a Luttinger liquid with two (charge and spin) linearly dispersing gapless modes, and there are two Fermi wavevectors associate with $\uparrow$ and $\downarrow$ fermions, whose magnitudes are determined individually by $N_{\uparrow}$ and $N_{\uparrow}$, which are separately conserved $[\underline{6}]$. In the presence of bosonic degrees of freedom and Bose-Fermi mixture as described by $H_{1 D}$ in Eq. (14), it was pointed out recently 22] that a new gapless mode may appear due to the condensation (or formation of an additional Luttinger liquid) of the bosons. This "decoupled spingapless phase" 22] is the 1D analog of the high-D phases with a Bose condensate discussed earlier. We show below that there exist 1D versions of Luttinger's theorem that completely determine the Fermi wavevectors in the absence of this new gapless mode, while in the presence of it (or in the decoupled spin-gapless phase) the Luttinger's theorem only gives a constraint on the Fermi wavevectors in a manner analogous to the high $\mathrm{D}$ cases discussed earlier.

Following Ref. 6, we consider a system with linear size $L$ and impose periodic boundary condition. We introduce twist operators that are appropriate to $H_{1 D}$ in Eq. (14), in spin and charge sectors respectively:

$$
\begin{aligned}
& U_{s}=\exp \left[i \frac{2 \pi}{L} \sum_{j} j\left(n_{j \uparrow}-n_{j \downarrow}\right)\right], \\
& U_{c}=\exp \left[i \frac{2 \pi}{L} \sum_{j} j\left(n_{j \uparrow}+n_{j \downarrow}+2 n_{j b}\right)\right] .
\end{aligned}
$$

It is straightforward to show that $U_{s, c}|0\rangle$ generates states with momenta

$$
\begin{aligned}
& k_{s}=\frac{2 \pi}{L}\left(N_{\uparrow}-N_{\downarrow}\right)=\frac{2 \pi}{L} N_{s}, \\
& k_{c}=\frac{2 \pi}{L}\left(N_{\uparrow}+N_{\downarrow}+2 N_{B}\right)=\frac{2 \pi}{L} N_{c},
\end{aligned}
$$

whose energies vanish as $1 / L$ in the limit $L \rightarrow \infty$. These gapless excitations at finite wavevectors correspond to combinations of $2 k_{F}$ excitations in Luttinger liquids [6].
In the absence of the new gapless mode due to the bosons, there are one spin mode and one charge mode that are gapless, and the two Fermi wavevectors can be uniquely determined by (17) and (18):

$$
2 k_{F \sigma}=\left(k_{c}+\sigma k_{s}\right) / 2=(\pi / L)\left(N_{c}+\sigma N_{s}\right),
$$

where $\sigma= \pm 1$ for $\uparrow / \downarrow$. On the other hand when there is a new gapless mode due to the bosons, there are one spin mode and two charge modes that are gapless; in this case there is an additional Fermi wavevector associated with this new charge mode (which may be interpreted as the Fermi wave vector of the condensed bosons). In this case Eqs. (17) and (18) can no longer determine all the Fermi wavevectors uniquely. However Eq. (17) still poses a constraint for the Fermi wavevectors that correspond to the fermions:

$$
2\left(k_{F \uparrow}-k_{F \downarrow}\right)=k_{s}=(2 \pi / L) N_{s} .
$$

These results are completely analogous to those obtained for high D systems.

It has been shown [26] recently that correlations in trapped 1D cold atom systems can be probed through interference experiments. In particular, it was found that the intensity of interference patterns can be used to directly measure the square of the equal time single particle Green's function [26]. For fermions, the Green's function is oscillatory with characteristic wave vector $k_{F}$. As a result $k_{F}$ can be extracted from such experiments, and our results above on 1D systems with Bose-Fermi mixture can be tested directly.

\section{QUANTUM PHASE TRANSITIONS}

We conclude this paper by presenting the field theories controlling the various quantum phase transitions and multicritical points in Fig. 1 in the following subsections. Some aspects of these theories were also discussed in Ref. 4 .

\section{1. $2 F S / 1 F S$}

We begin with the transition between the 2FS and 1FS phases at large $h$. Here a Fermi surface gradually shrinks to zero volume, in the absence of any bosonic condensate. Denoting the fermionic quasiparticle excitations of this small Fermi surface by $\psi$, and following the analysis of the dilute Fermi gas critical point in Chapter 11 of Ref. 27, we initially guess that the critical theory is given by the free fermion form

$$
S_{\psi}=\int d^{d} x d \tau \psi^{\dagger}\left(\frac{\partial}{\partial \tau}-\frac{1}{2 m_{\psi}} \nabla^{2}+s\right) \psi,
$$

where $\tau$ is imaginary time, and $s$ is the parameter which tunes across the transition. In this case, the quantum 
critical point is at $s=0$, and the small Fermi surface is present for $s>0$. The simplest four Fermi interaction must involve two gradients because of the Pauli principle, and is of the form $\sim\left(\psi^{\dagger} \nabla \psi\right)^{2}$. A scaling analysis with dynamic exponent $z=2$ [27] shows that the dimension of this term is $-d$, and so such interactions are always irrelevant. However, here we have a second large Fermi surface present, and we have to allow for long-range interactions induced by the fluctuations of this Fermi surface. The oscillations of this second Fermi surface will couple to $\psi^{\dagger} \psi$, and integrating these out following Hertz 28] we obtain the following long-range interaction (for $d>1$ )

$$
\lambda \int_{\mathbf{k}, \omega}\left[\psi^{\dagger} \psi\right]_{\mathbf{k}, \omega} \frac{|\omega|}{k}\left[\psi^{\dagger} \psi\right]_{-\mathbf{k},-\omega}
$$

Power-counting with $z=2$ shows that $\lambda$ has scaling dimension $1-d$, and so is irrelevant. The $d=1$ case requires special treatment, and we will not consider it here.

\section{2. $2 F S+B E C / 1 F S+B E C$}

This is just like the 2FS/1FS transition above, except that a background Bose condensate is present. Fluctuations in the superflow of this condensate induce longrange interactions, which could be important at the critical point. With $\varphi$ being the phase of the condensate, the coupling to the $\psi$ excitations of the current-current type [8]: $\partial_{i} \varphi\left(\psi^{\dagger} \partial_{i} \psi-\partial_{i} \psi^{\dagger} \psi\right)$. Integrating out the $\varphi$, we now generate the interaction

$$
\begin{aligned}
& \int_{\mathbf{k}_{1}, \mathbf{k}_{2}, \mathbf{q}, \epsilon_{1}, \epsilon_{2}, \omega} \psi^{\dagger}\left(\mathbf{k}_{1}+\mathbf{q}, \epsilon_{1}+\omega\right) \psi\left(\mathbf{k}_{1}, \epsilon_{1}\right) \\
& \times \frac{\left(\mathbf{k}_{1} \cdot \mathbf{q}\right)\left(\mathbf{k}_{2} \cdot \mathbf{q}\right)}{q^{2}} \psi^{\dagger}\left(\mathbf{k}_{2}-\mathbf{q}, \epsilon_{2}-\omega\right) \psi\left(\mathbf{k}_{2}, \epsilon_{2}\right) .
\end{aligned}
$$

Again, simple power-counting shows that this term has scaling dimension $-d$, and so is irrelevant. So the critical theory remains the free fermion theory $S_{\psi}$.

\section{3. $B E C / 1 F S+B E C$}

The considerations for this are identical to the 2FS+BEC/1FS+BEC transition above, as both involve the disappearance of a Fermi surface in the presence of bosonic condensate.

\section{4. $B E C / 2 F S+B E C$}

This is a novel transition involving the simultaneous appearance of two Fermi surfaces at a spherical minimum in the fermionic quasiparticle dispersion. This was considered in a separate paper [11] for the case of nonsingular (or short-range) fermionic interactions. The superflow fluctuations will also introduce here an interaction between the fermions of the form in Eq. (23). Because we are now interested in fermions at large momentum Fermi surface, the $\mathbf{k}_{1,2}$ in Eq. (23) will be replaced by $k_{0} \mathbf{n}$, where $k_{0}$ is the minimum of the fermion dispersion (that forms a sphere; it also becomes the Fermi wavevector once fermions start to populate these modes), and $\mathbf{n}$ is its normal direction. This changes the power-counting in the scaling dimension of this coupling. We discuss the renormalization group (RG) flow of such anisotropic, long-range interactions below.

As discussed in Ref. 11, the relevant fermionic modes near the critical point are those within a thin spherical shell in momentum space: $k_{0}-\Lambda<|\mathbf{k}|<k_{0}+\Lambda$, where $\Lambda \ll k_{0}$ is a cutoff scale. Phase space restriction thus divide the possible scattering processes into two classes, which need to be treated separately:

(i) The Cooper channel, in which $\mathbf{k}_{1} \approx-\mathbf{k}_{2}$, and the momentum transfer $q$ can be as large as $O\left(k_{0}\right)$. In this channel the interaction of Eq. (223) simply reduces to those studied in Ref. 11, and the results there can be applied directly to the present case.

(ii) Forward scattering channel, in which $\left|\mathbf{k}_{1}+\mathbf{k}_{2}\right| \gg \Lambda$, and the magnitude of momentum transfer is constrained to be $|\mathbf{q}| \lesssim \Lambda$. In the absence of long-range interactions, we can then take the limit $|\mathbf{q}| \rightarrow 0$, and assume that there is no further dependence on its orientation $\mathbf{q} /|\mathbf{q}|$. However, with the anisotropic long-range interactions induced by superflow fluctuations in Eq. (23), the limit of vanishing $|\mathbf{q}|$ can be taken, but there remains a residual dependence on $\mathbf{q} /|\mathbf{q}|$. Here we perform an RG analysis of the most general interaction of fermions on the Fermi surface dependent upon $\mathbf{q} /|\mathbf{q}|$, and for simplicity focus on two-dimensional (2D) systems; the situation in 3D is expected to be similar qualitatively. Such an interaction takes the following form in $2 \mathrm{D}$ :

$$
V\left(\mathbf{k}_{1}, \mathbf{k}_{2}, \mathbf{q}\right)=\sum_{n} V_{n}(\theta) \cos \left(n \phi_{\mathbf{q}}\right),
$$

where $\theta$ is the angle between $\mathbf{k}_{1}$ and $\mathbf{k}_{2}$, and $\phi_{\mathbf{q}}$ is the angle between $\mathbf{q}$ and $\mathbf{k}_{1}+\mathbf{k}_{2}$. For short-range interactions only the $n=0$ term exists, and this case was analyzed in Ref. 11. The interaction in Eq. (23) generates a term with $n=2$. We now perform a one-loop RG analysis 11] of the interactions in Eq. (24), and obtain the following flow equation:

$$
\frac{d V_{n}(\theta)}{d \log s}=\delta_{n, 0} \beta_{0}[V]
$$

where $s$ is the cutoff rescaling factor and

$$
\beta_{0}[V]=\frac{-2 m^{*}}{\pi^{2}|\sin \theta|} \int_{-1}^{1} \frac{\left|\sum_{n} V_{n}(\theta) \cos [n \phi(t)]\right|^{2}}{1+t^{2}} d t
$$

in which $\phi(t)=\arctan [(t-\cos \theta) / \sin \theta]-\theta / 2$, and $m^{*}$ is a parameter that parameterize the fermion dispersion 
near the minimum: $\epsilon(k)=\left(k-k_{0}\right)^{2} /\left(2 m^{*}\right)$. It is clear from Eq. (25) that only $V_{0}$ gets renormalized under RG transformations, while interactions with $n \neq 0$ remains marginal. Physically this is because the renomalization is due to multiple virtual scattering processes whose momentum transfers are of order the cutoff $\Lambda$, resulting in renomalization that is not sensitive to the net momentum transfer much smaller than $\Lambda$ at low-energy. Thus the $n \neq 0$ quasiparticle interactions remain at their bare values, and are finite and non-universal in the low-energy limit. For the case of the quantum phase transition of interest in this subsection, the $n=2$ interaction in Eq. (23) therefore acquires no loop corrections. Furthermore, in the presence of $V_{n \neq 0}, V_{0}$ will always be driven negative and flow away, even if it is initially positive (or repulsive). The situation was very different when only the short-range interaction $V_{0}$ was present; in that case it was found 11] that for repulsive interactions, it flows to zero logarithmically, which leads to an effective quasiparticle interaction that takes a universal form in the low-energy limit due to this renormalization. Here, we observe from Eq. (26) that the $n=2$ interactions leads to a run-away flow of $V_{0}$ that has no fixed point. Physically, this suggests that either the transition from BEC to $2 \mathrm{FS}+\mathrm{BEC}$ is first order, or that the instability of the BEC in this case is toward a state with other symmetry properties, like the FFLO state. We note that related observations were made in Ref. 8 based on quite different considerations.

\section{5. $1 F S / 1 F S+B E C$}

This involves the appearance of a BEC in the presence of Fermi surface. The BEC onset is described by the dilute Bose gas theory discussed in Chapter 11 of Ref. 27, and in Ref. 29. The action is

$$
\begin{gathered}
S_{b}=\int d^{d} x \int d \tau\left[b^{\dagger} \frac{\partial b}{\partial \tau}-\frac{1}{2 m_{b}} b^{\dagger} \nabla^{2} b+s|b|^{2}\right. \\
\left.+\frac{u}{2}|b|^{4}\right],
\end{gathered}
$$

where $s$ is again the tuning parameter, and the quantum critical point is at $s=0$. The scaling dimension of the quartic coupling $u$ is $(2-d)$, and so is formally irrelevant for $d>2$. However, this coupling is dangerously irrelevant, because a $u>0$ is required to stabilize the system for $s<0$. Couplings to Fermi surface fluctuations will induce a long-range interaction analogous to Eq. (22) among the bosons

$$
\lambda \int_{\mathbf{k}, \omega}\left[|b|^{2}\right]_{\mathbf{k}, \omega} \frac{|\omega|}{k}\left[|b|^{2}\right]_{-\mathbf{k},-\omega} .
$$

Just as in Eq. (22) however, power-counting near a $z=2$ transition shows that this coupling has scaling dimension $1-d$, and so is irrelevant.

$$
\text { 6. } 2 F S / 2 F S+B E C
$$

The considerations for this are nearly identical to the 1FS/1FS+BEC transition discussed above. Here, we also have to consider the decay of a boson into two fermions, one each on the respective Fermi surfaces. However, this is a low energy process only at finite wavevectors which connect the the two Fermi surfaces. Consequently it can be safely neglected for the low momentum $b$ critical theory.

\section{Multicritical point $M$}

The theory for this point is essentially the direct sum of the theories for the two transition lines which intersect at M. There is a bosonic critical mode, $b$, described by $S_{b}$, and a fermionic critical mode $\psi$, described by $S_{\psi}$. These two critical modes can interact with each other via the contact term

$$
g \int d^{d} x d \tau|b|^{2} \psi^{\dagger} \psi
$$

and $g$ has dimension $(2-d)$. So it will have appreciable effects in $d=2$ which can be computed along the lines of Ref. 29. The coupling $g$ will also be very important in $d=1$.

\section{Multicritical point $L$}

This is a 'Lifshitz' point, which was considered in Ref. 8 , albeit with a different perspective. This is the point where the fermionic dispersion minimum moves from $\mathbf{k}=0$ to a non-zero $k$. Consequently, the dispersion at small $\mathbf{k}$ is $\sim k^{4}$, and the multicritical theory has $z=4$ :

$$
S_{\psi, L}=\int d^{d} x d \tau \psi^{\dagger}\left(\frac{\partial}{\partial \tau}+\nabla^{4}\right) \psi
$$

The simplest four Fermi interaction is again $\sim\left(\psi^{\dagger} \nabla \psi\right)^{2}$, and a scaling analysis with $z=4$ shows that this has scaling dimension $(2-d)$. The superflow fluctuations will also induce a long range interaction as in Eq. (23), and this again has scaling dimension $(2-d)$. So these interactions are irrelevant for $d>2$, while a detailed analysis of logarithmic corrections is necessary in $d=2$. We do not present this here, but it can be carried out as in Ref. 29 .

\section{Acknowledgments}

This work was supported by National Science Foundation grants DMR-0537077 (SS), DMR-0225698 (KY), and a Florida State University Research Foundation Grant (KY). 
[1] M. W. Zwierlein, A. Schirotzek, C. H. Schunck, and W. Ketterle, Science 311, 492 (2006).

[2] G. B. Partridge, W. Li, R. I. Kamar, Y. Liao, and R. G. Hulet, Science 311, 503 (2006).

[3] C. A. Regal, M. Greiner, S. Giorgini, and D. S. Jin, Phys. Rev. Lett. 95, 250404 (2005).

[4] S. Powell, S. Sachdev and H. P. Büchler, Phys. Rev. B 72, 024534 (2005).

[5] P. Coleman, I. Paul, and J. Rech, Phys. Rev. B 72, 094430 (2005).

[6] M. Yamanaka, M. Oshikawa, and I. Affleck, Phys. Rev. Lett. 79, 1110 (1997).

[7] C.-H. Pao, Shin-Tza Wu, and S.-K. Yip, cond-mat/0506437

[8] D. T. Son and M. A. Stephanov, cond-mat/0507586

[9] D. E. Sheehy and L. Radzihovsky, cond-mat/0508430

[10] K. Yang, cond-mat/0508484

[11] K. Yang and S. Sachdev, cond-mat/0511641

[12] M. Haque and H. T. C. Stoof, cond-mat/0601321

[13] P. Fulde and R. A. Ferrell, Phys. Rev. 135, A550 (1964).

[14] A. I. Larkin and Yu. N. Ovchinnikov, Sov. Phys. JETP 20, 762 (1965).

[15] G. Sarma, J. Phys. Chem. Solids 24, 1029 (1963).

[16] W. V. Liu and F. Wilczek, Phys. Rev. Lett. 90, 047002
(2003); M. M. Forbes, E. Gubankova, W. V. Liu, and F. Wilczek, Phys. Rev. Lett. 94, 017001 (2005).

[17] P. F. Bedaque, H. Caldas, and G. Rupak, Phys. Rev. Lett. 91, 247002 (2003).

[18] J. Carlson and S. Reddy, Phys. Rev. Lett. 95, 060401 (2005).

[19] J. M. Luttinger and J. C. Ward, Phys. Rev. 118, 1417 (1960).

[20] M. Potthoff, cond-mat/0406671

[21] J. M. Luttinger, Phys. Rev. 119, 1153 (1960).

[22] D. E. Sheehy and L. Radzihovsky, Phys. Rev. Lett. 95, 130401 (2005).

[23] R. Citro and E. Origanic Phys. Rev. Lett. 95, 130402 (2005).

[24] H. A. Radovan et al., Nature 425, 51 (2003).

[25] A. Bianchi et al., Phys. Rev. Lett. 91, 187004 (2003).

[26] A. Polkovnikov, E. Altman, and E. Demler, cond-mat/0511675

[27] S. Sachdev, Quantum Phase Transitions, Cambridge University Press, Cambridge, 1999.

[28] J. A. Hertz, Phys. Rev. B 14, 1165 (1976).

[29] S. Sachdev and E. Dunkel, cond-mat/0510365 